Comparison of material response in fused silica and KDP following exit surface laser-induced breakdown

S. G. Demos, R. A. Negres, R. N. Raman, A. M. Rubenchik, M. D. Feit

October 22, 2013

SPIE Laser Damage 2013

Boulder, CO, United States

September 21, 2013 through September 25, 2013 
This document was prepared as an account of work sponsored by an agency of the United States government. Neither the United States government nor Lawrence Livermore National Security, LLC, nor any of their employees makes any warranty, expressed or implied, or assumes any legal liability or responsibility for the accuracy, completeness, or usefulness of any information, apparatus, product, or process disclosed, or represents that its use would not infringe privately owned rights. Reference herein to any specific commercial product, process, or service by trade name, trademark, manufacturer, or otherwise does not necessarily constitute or imply its endorsement, recommendation, or favoring by the United States government or Lawrence Livermore National Security, LLC. The views and opinions of authors expressed herein do not necessarily state or reflect those of the United States government or Lawrence Livermore National Security, LLC, and shall not be used for advertising or product endorsement purposes. 


\title{
Comparison of material response in fused silica and KDP following exit surface laser- induced breakdown
}

\author{
Stavros G. Demos*, Raluca A. Negres, Rajesh N. Raman, Alexander M. Rubenchik, Michael D. Feit \\ Lawrence Livermore National Laboratory, 7000 East Avenue, Livermore, CA, USA 94550
}

\begin{abstract}
Crater formation that accompanies laser-induced damage is the result of material ejection following the rapid, localized heating to temperatures on the order of $1 \mathrm{eV}$. The objective of this work is to compare the material ejection behavior in fused silica and KDP crystals as captured using time-resolved shadowgraphy. These two materials are of fundamental importance in ICF class laser systems but they also represent materials with significantly different physical properties. We hypothesize that these different properties can affect the material ejection process.
\end{abstract}

Keywords: Fused silica, $\mathrm{SiO}_{2}, \mathrm{KDP}, \mathrm{DKDP}$, laser-induced damage, laser-induced breakdown, material ejection, laser ablation

\section{INTRODUCTION}

Laser-induced damage in optical materials for high power nanosecond laser systems arises from localized energy deposition into the material due to the presence of pre-existing light absorbing defects [1-6]. Laser damage on the surface of optical materials is associated with a cascade of material response effects that include plasma formation, expansion and confinement, high pressure and temperature of the affected material, surface swelling, gaseous material ejection, generation and propagation of shockwave and stress waves, particle ejection, mechanical damage of adjacent material including cracking and pulverization, as well as elastic and plastic deformation leading to densification [7]. These effects ultimately lead to the formation of a crater on the surface that involves modified material having a high concentration of defects [8-12]. Since a detailed theoretical description of this multi-component process is currently not feasible, our approach is to advance knowledge by performing time-resolved experiments with adequate spatial and temporal resolution.

Fused silica and potassium dihydrogen phosphate (KDP and its deuterated analog, DKDP) are key optical materials in ICF class laser systems $[1,5]$. They both exhibit limitations in performance under high fluence irradiation associated with laser-induced damage. Under the current operational conditions, these two materials are exposed to similar laser fluences. However, their material properties are distinctly different [13]. Fused silica is amorphous while KDP is a tetragonal crystal. Fused silica has very low thermal expansion coefficient and a high temperature has to be reached before evaporation is significant. KDP has a thermal expansion coefficient about 50 times larger than that of silica and a very low melting temperature so evaporation can be significant at much lower temperatures. Therefore, one expects the dynamic response in each material following ns pulse laser energy deposition to be, at least in part, influenced by their respective material properties.

To address the impact of these different material properties, we have extended our previous studies of the material behavior following exit surface damage in fused silica with corresponding experiments performed on KDP. The results demonstrate a very different early stage behavior where the evaporation and ejection of material exhibit different kinetics between the two materials. After this initial period, an ejected material jet forms in KDP that is similar to that observed in fused silica. Furthermore, the temporal duration of the material ejection process is much longer in KDP, extending to more than $100 \mu$ s from the initiation laser pulse compared to about $20 \mu$ s measured in fused silica. Side-byside comparison of the dynamic response in these materials following ns pulsed laser energy deposition reveals mechanisms by which the intrinsic properties of the material affect the material ejection and the final morphology of the damage site.

*Corresponding author: demos1@1lnl.gov 


\section{EXPERIMENTAL DESIGN}

Experiments were performed using time-resolved shadowgraphy microscopy configured to capture two transient images per event. The delay of each image with respect to the pump pulse was independently adjusted. A schematic depiction of the experimental setup is shown in Figure 1. The time-resolved microscope system has been described in detail elsewhere [14]. In brief, damage was induced on the exit (rear) surface of fused Silica and z-cut KDP samples by exposure to a focused $\left(\approx 50 \mu \mathrm{m} \mathrm{1} / \mathrm{e}^{2}\right.$ diameter at the surface location) laser beam using a $5-\mathrm{cm}$ focal length lens with focal point located about $1 \mathrm{~cm}$ beyond the sample's exit surface. The pump laser operated at 1064-nm, $10 \mathrm{~ns}$ FWHM (full-width-half-maximum intensity) and the estimated peak fluence on the exit surface was on the order of $10^{3} \mathrm{~J} / \mathrm{cm}^{2}$. The image plane of the microscope was orthogonal to the sample's surface and parallel to the direction of pump beam propagation. The side of the sample was illuminated with two spatially overlapped, orthogonally polarized $532 \mathrm{~nm}, 4.5$ ns FWHM probe laser pulses. A composite $5 \mathrm{X}$ zoom and $2 \mathrm{X}$ objective lens system was used to collect the dual-probe signal traversing the ejected material volume; this signal was subsequently passed through a 532-nm narrowband filter and separated into its constituent polarization components using a polarizing beam-splitter. Thus each component arising from a different probe captures the dynamics of the ejected material at a different pump-probe delay time. Images from each probe beam were recorded by separate charge-coupled device (CCD) cameras.

The top inset shown in Figure 1 summarizes the main processes involved during exit surface laser-induced damage including the material ejection process and the formation of a shockwave in the air. These two components are the focus of this investigation. In particular, the time-resolved imaging system is designed to capture the microscopic particle ejection and the propagation of the shockwave. The spatial resolution of the system is reduced to about $2 \mu \mathrm{m}$ (static resolution) in order to increase the field of view to better capture the differences in the material ejection process between the two materials. A detailed description of the material ejection process in fused silica under $355 \mathrm{~nm}, 3 \mathrm{~ns}$ laser excitation with one order of magnitude lower fluences than those used in this work has been provided elsewhere $[15,16]$.

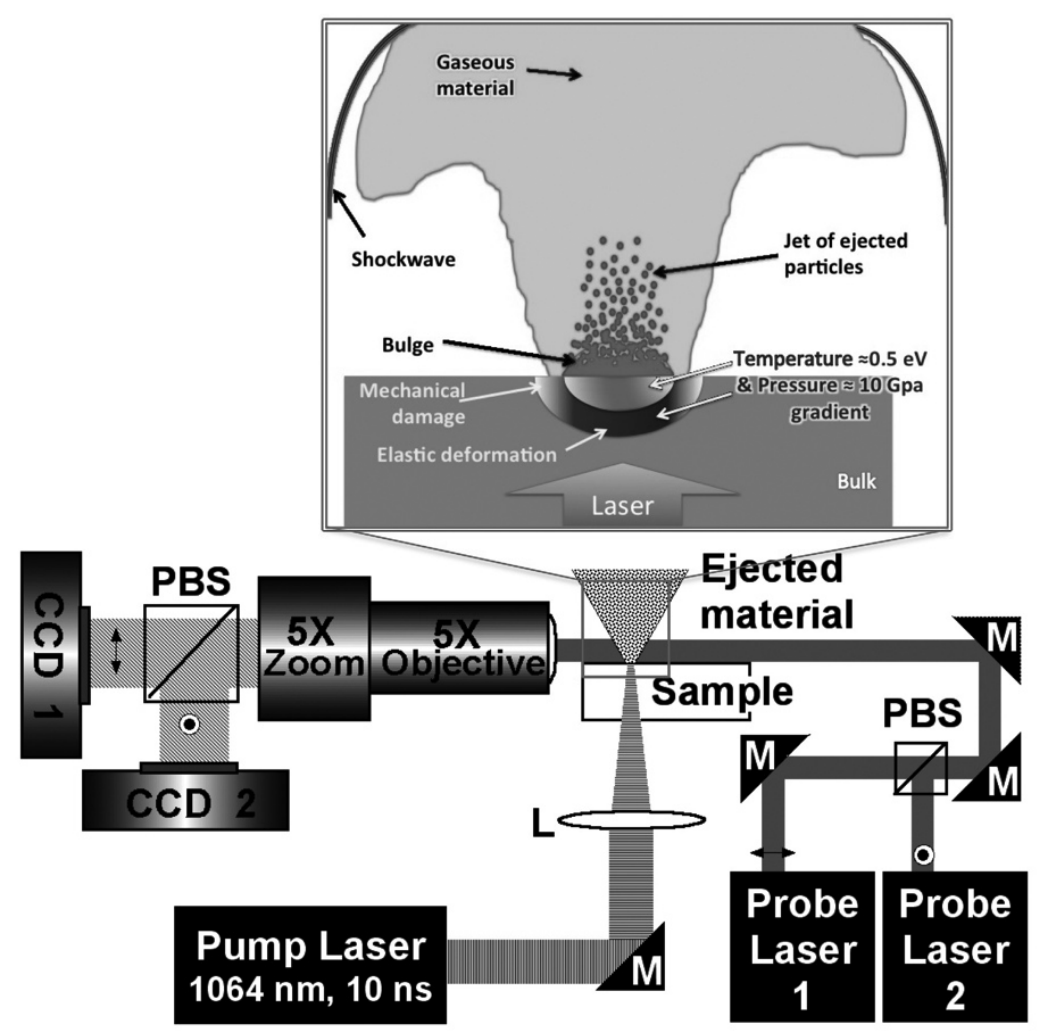

Figure 1. Schematic diagram of the main components of the experimental system. Top inset represents a depiction of the main processes involved during exit surface laser-induced damage. 


\section{EXPERIMENTAL RESULTS}

Figure 2 shows time-resolved images of the material ejection process in fused silica and KDP at $150 \mathrm{~ns}$ and $450 \mathrm{~ns}$ delay. The location of the shockwave propagating in air is denoted by an arrow. The fairly similar expansion of the shockwave in the two materials indicates that the initial energy deposited was approximately the same during the individual laser-induced breakdown events. For the case of exit surface damage in fused silica (top images in Figure 2), the ejected particles formed a well-defined jet which is similar to that observed under excitation at about $40 \mathrm{~J} / \mathrm{cm}^{2}$ at $3 \omega$, despite the very different excitation conditions (detailed elsewhere, $[15,16]$ ). In contrast, exit surface damage in KDP (bottom images in Figure 2) produces an initial conical shell pattern of ejected particles. The speed of the ejected particles in KDP (i.e., location of the particle jet front) is also slower than that observed in fused silica by a factor of about 2 .
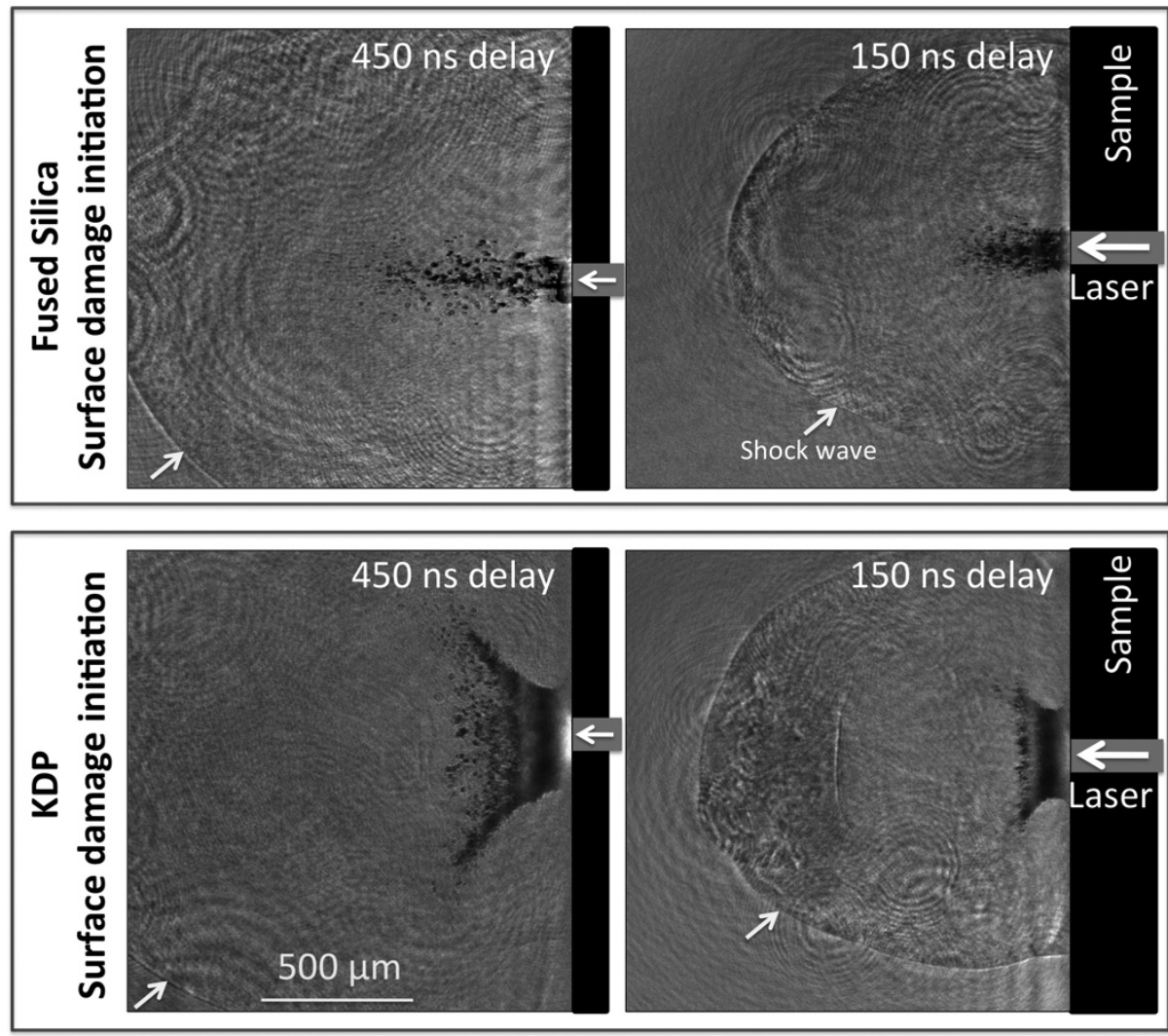

Figure 2. Comparison of early material response following exit surface breakdown in fused silica (top) and KDP (bottom):

paired images of the same breakdown event at $150 \mathrm{~ns}$ and $450 \mathrm{~ns}$ pump-probe delay times, respectively.

At later delays, the conical shell pattern of ejected particles in KDP is followed by a regular jet, similar to that observed in fused silica. This behavior is captured in the time-resolved images shown in Figure 3 illustrating the evolution of the particle ejection at $2.5 \mu \mathrm{s}, 10.5 \mu \mathrm{s}, 30.5 \mu \mathrm{s}$ and $200 \mu \mathrm{s}$ delays, respectively. Specifically, the image captured at $2.5 \mu \mathrm{s}$ delay shows the conical expansion of the early ejected particles; however, at the base, the jet appears to significantly narrow. This transition from conical ejection to conventional ejection within a narrow angle from the direction vertical to the surface is best depicted by the image captured at $10.5 \mu$ s delay shown in Figure 3. In particular, this image shows a conventional narrow jet of particles ejected from the surface of KDP, in contrast with the initial conical shaped jet observed at early delays. In addition, this image captured at $10.5 \mu$ s delay shows that the width of the jet is wider near the surface. This is attributed to the onset of ejection of mechanically damaged material from the region surrounding the original site of laser energy deposition. The particles originating from this area appear larger and have the form of flakes and chips of mechanically (impact) damaged KDP material. The image captured at $30.5 \mu$ s delay show the propagation of these larger ejected particles along a path that is also vertical to the surface. The image captured at $200 \mu$ s delay shows 
the last group of particles ejected from the surface that have speeds of about $6 \mathrm{~m} / \mathrm{sec}$ (for the particles farther from the surface) or less.

A different behavior was observed in KDP during exit surface damage initiation with lower fluences, on the order of 500 $\mathrm{J} / \mathrm{cm}^{2}$. The results shown in Figure 4 are representative of this latter behavior. Specifically, the shockwave formed in the air is clearly visible but propagates at a lower speed (by a factor of about 0.4) compared to the case depicted in Figure 2 for exit surface damage in fused silica. In addition, the conically expanding jet of particles observed at early delay times (Figure1) is absent. In fact, only very few particles are visible or often no particles at all. Our interpretation of this behavior is that the energy deposition is taking place below the surface, thus limiting the initial ejection of particles. However, this process is taking place close enough to the surface so that the surface swelling can support the generation of the shockwave. It is likely that gaseous material is also produced during this early phase (not visible with this method), which can contribute to the generation and enhancement of the shockwave. In the images shown in Figure 4 captured at 150 and 450 ns delays, respectively, ejected particles are barely visible (a much smaller number of them) and, at later delays, they are forming a convectional jet (see images in Figure 4 captured at 10.5 and $30.5 \mu \mathrm{s}$, respectively). This latter effect may be attributed to the eruption of the sub-surface damage site followed by relaxation of the affected material in part via the production of ejected particles.

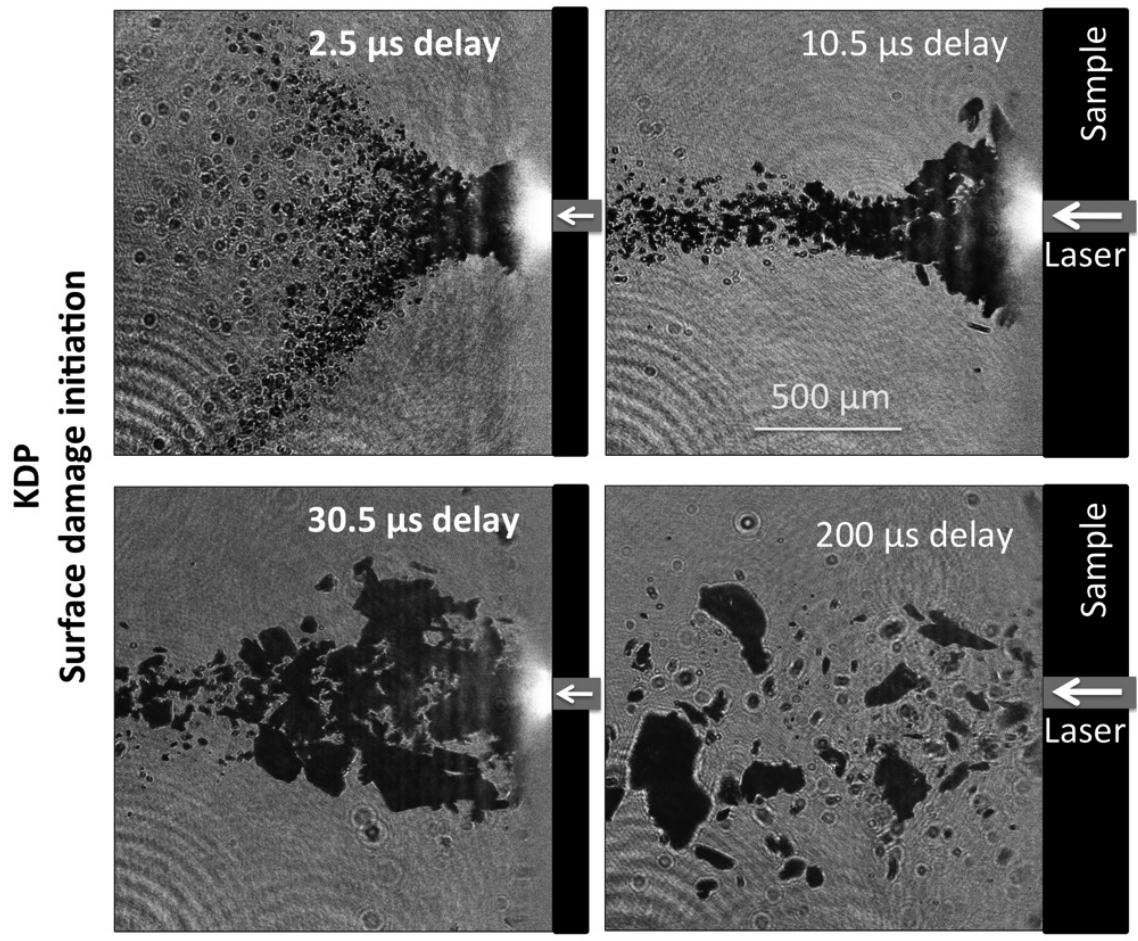

Figure 3. Evolution of material ejection in KDP following surface damage initiation. All images are from different breakdown events. 


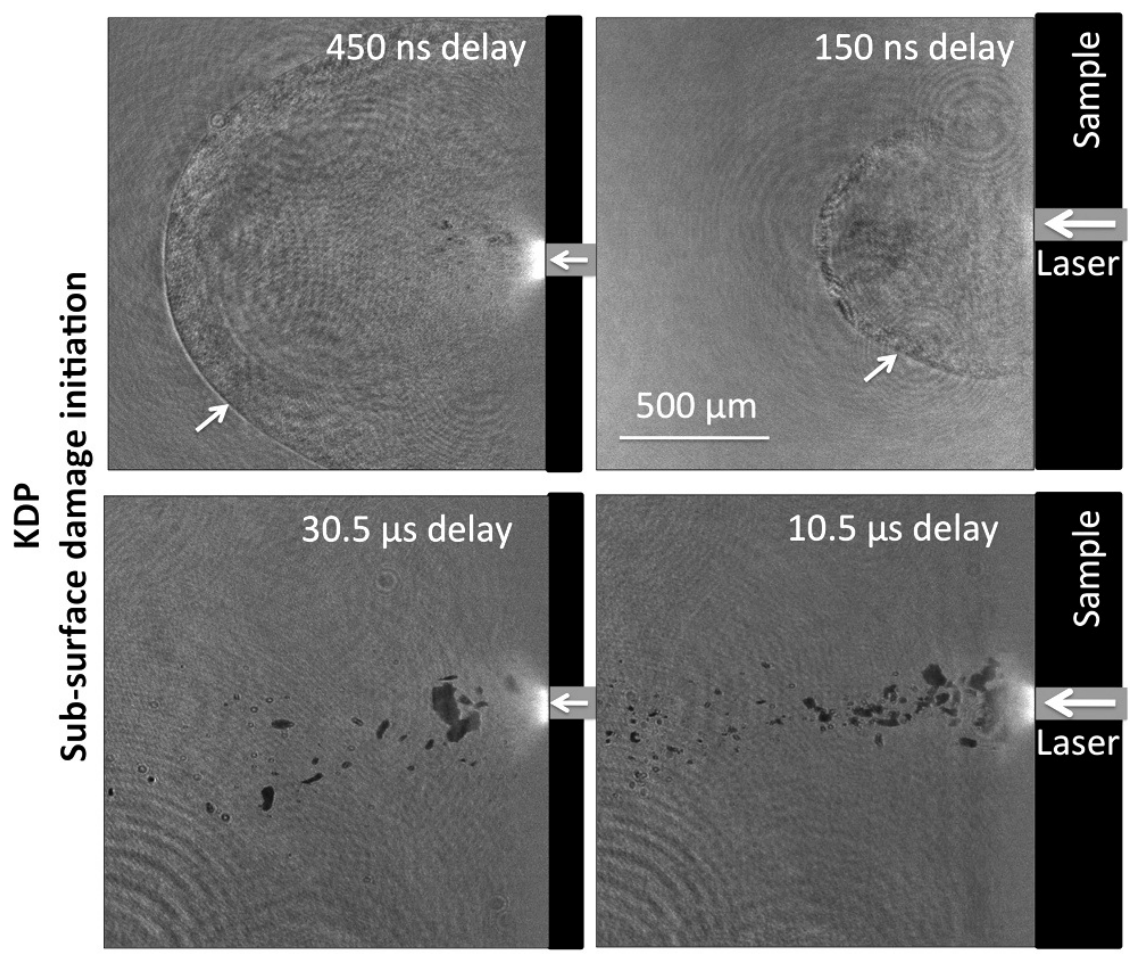

Figure 4. Evolution of material ejection in KDP following sub-surface damager initiation. All images are from different breakdown events.

\section{DISCUSSION}

Based on the kinetics of the ejected material in KDP, damage initiation can be separated into two types, surface-initiated and subsurface-initiated. Both of these salient behaviors in KDP may be assigned to the expansion of the partially confined plasma region followed by explosive relaxation including ejection of gaseous material and micro-scale particles. This process follows the initial generation of a shockwave of the laser-heated volume, which may be driven, by surface swelling (mostly for the subsurface damage initiation case) and an initial evaporation of affected material near the surface. Additional details on these processes involved in the early phase of material ejection during laser damage will be discussed in detail elsewhere.

Due to the low boiling temperature of $\mathrm{KDP}-400 \mathrm{C}^{\circ}$ in $\mathrm{KDP}$ vs. $\sim 2300 \mathrm{C}^{\circ}$ in $\mathrm{SiO}_{2}$, the initial response of the affected superheated material is quite different. In particular, the ejection of material in KDP takes place mainly via the production of gaseous material while, in contrast, $\mathrm{SiO}_{2}$ can support production of liquid droplets from early delays. As a result, only the peripheral regions of the heated region in DKDP where the temperature is considerably lower can support formation of micro-scale particles. These particles are ejected in the presence of the expanding gaseous material from the central region. The combined effect is captured with the observation at early delays of ejection in KDP of particles in a conical pattern, as shown in Figure 2.

At later delays, the adjacent material to the damage spot is heated up to high temperature. The thermal conduction is low in both, KDP and fused silica and probably evaporation remains the main cooling mechanism in both materials. For fused silica the evaporation stops when the temperature drops below $2300 \mathrm{C}^{\circ}$ while in KDP evaporation stops at $\mathrm{T} \sim 400$ $\mathrm{C}^{\circ}$ (and melting at $250 \mathrm{C}^{\circ}$ ). Therefore, the evaporation in KDP will probably continue to much longer delays along with the ejection of liquid droplets.

A jet propagating vertical to the surface is observed in the transient images captured at later delays, similar to that observed in fused silica, indicating similar underlying mechanisms. Since the ejected particles in KDP are at lower temperature, a relatively larger fraction of them are due to mechanically damaged material. Stored elastic energy may be in part responsible for the kinetic energy of the ejected particles. 
The transient images shown in Figure 3 demonstrate that the material removal in KDP extends well outside the region of the incident pump laser beam, in contrast with the behavior observed in $\mathrm{SiO}_{2}$. The lower fracture toughness of KDP may be responsible for this behavior $\left(0.1 \mathrm{MPa} \cdot \mathrm{m}^{1 / 2}\right.$ in $\mathrm{KDP}$ vs. $0.75 \mathrm{MPa} \cdot \mathrm{m}^{1 / 2}$ in $\mathrm{SiO}_{2}$ at room temperature) giving rise to a wider region of mechanically pulverized material. This weakly attached, mechanically damaged material may govern material ejection at later delays and continues for a longer period of time in KDP than in $\mathrm{SiO}_{2}$; these latter effects may be due to reflected waves and/or surface motion resulting from the recoil pressure applied to the material following laser energy deposition.

Comparison of the results in KDP and fused silica indicates the importance of melting and evaporation temperatures in determining the material response when both materials are subjected to similar initial temperatures and pressures during a damage event. The results also suggest temperature differences and pressure gradients within the affected material volume play an important role in the material ejection process.

\section{ACKNOWLEDGEMENTS}

This work was performed under the auspices of the U.S. Department of Energy by Lawrence Livermore National Laboratory under Contract DE-AC52-07NA27344. LLNL-PROC-644961

\section{REFERENCES}

[1] H. Bercegol, A. Boscheron, J. -M. Di-Nicola, E. Journot, L. Lamaignere, J. Neauport, and G. Raze, "Laser damage phenomena relevant to the design and operation of an ICF laser driver,", J/ Phys. Conf. Ser. 112, 032013 (2008).

[2] A. K. Burnham, M. Runkel, M. D. Feit, A. M. Rubenchik, R. L. Floyd, T. A. Land, W. J. Siekhaus, and R. A. Hawley-Fedder, "Laser-induced damage in deuterated potassium dihydrogen phosphate," Appl. Opt. 42, 5483-5495 (2003).

[3] S. G. Demos, M. Staggs, "Application of fluorescence microscopy for noninvasive detection of surface contamination and precursors to laser-induced damage" Appl. Opt., 41, 1977-1983, 2002

[4] C. W. Carr, H. B. Radousky, A. M. Rubenchik, M. D. Feit, and S. G. Demos, "Localized dynamics during laserinduced damage in optical materials," Phys. Rev. Lett. 92, 087401 (2004).

[5] N. P. Zaitseva, J. J. DeYoreo, M. R. Dehaven, R. L. Vital, K. E. Montgomery, M. Richardson, and L. J. Atherton, "Rapid growth of large-scale $(40-55 \mathrm{~cm})$ KH2PO4 crystals," J. Cryst. Growth 180, 255-262 (1997).

[6] C. Maunier, P. Bouchut, S. Bouillet, H. Cabane, R. Courchinoux, P. Defossez, J. C. Poncetta, and N. FerriouDaurios, "Growth and characterization of large KDP crystals for high power lasers," Opt. Mater. 30, 88-90 (2007).

[7] S. G. Demos, R. A. Negres, R. N. Raman, A. M. Rubenchik, and M. D. Feit, "Material response during nanosecond laser induced breakdown inside of the exit surface of fused silica," Laser Photonics Rev. 7, 444-452 (2013).

[8] A. D. Walser, S. G. Demos, M. Etienne, and R. Dorsinville, "Nonlinear optical absorption in laser modified regions of fused silica substrates," Opt. Commun. 240, 417-421 (2004).

[9] J. Wong, J. L. Ferriera, E. F. Lindsey, D. L. Haupt, I. D. Hutcheon, and J. H. Kinney, "Morphology and microstructure in fused silica induced by high fluence ultraviolet $3 \omega(355 \mathrm{~nm})$ laser pulses," J. Non-cryst. Solids $352,255-272$ (2006).

[10] S. Juodkazis, M. Watanabe, H. B. Sun, S. Matsuo, J. Nishii, and H. Misawa, "Optically induced defects in vitreous silica," Appl. Surf. Sci. 154, 696-700 (2000).

[11] S. O. Kucheyev and S. G. Demos, "Optical defects produced in fused silica during laser-induced breakdown," Appl. Phys. Lett. 82, 3230-3232 (2003).

[12] S. G. Demos, M. Staggs, K. Minoshima, and J. Fujimoto, "Characterization of laser induced damage sites in optical components," Opt. Express 10, 1444-1450 (2002).

[13] Handbook of Optical Materials, ed. M. J. Weber, CRC press (2002).

[14]R. N. Raman, R. A. Negres, and S. G. Demos, "Time-resolved microscope system to image material response following localized laser energy deposition: exit surface damage in fused silica as a case example,” Opt. Eng. 50, 013602 (2011).

[15] R. N. Raman, R. A. Negres, and S. G. Demos, "Kinetics of ejected particles during breakdown in fused silica by nanosecond laser pulses," Appl. Phys. Lett. 98, 051901 (2011).

[16] R. N. Raman, S. Elhadj, R. A. Negres, M. J. Matthews, M. D. Feit, and S. G. Demos, "Characterization of ejected fused silica particles following surface breakdown with nanosecond pulses," Opt. Express 20, 27708-27724 (2012). 